\title{
Centrality Dependence of Multiplicity, Transverse Energy, and Elliptic Flow from Hydrodynamics
}

\author{
P.F. Kolb ${ }^{a, b}$, U. Heinz ${ }^{a}$, P. Huovinen ${ }^{c}$, K.J. Eskola ${ }^{d, e}$, and K. Tuominen ${ }^{d}$ \\ ${ }^{a}$ Department of Physics, The Ohio State University, 174 West 18th Avenue, Columbus, OH 43210, USA \\ ${ }^{b}$ Institut für Theoretische Physik, Universität Regensburg, D-93040 Regensburg, Germany \\ ${ }^{c}$ Lawrence Berkeley National Laboratory, Berkeley CA 94720, USA \\ ${ }^{d}$ Department of Physics, University of Jyväskylä, P.O. Box 35, FIN-40351 Jyväskylä, Finland \\ ${ }^{e}$ Helsinki Institute of Physics, P.O. Box 64, FIN-00014 University of Helsinki, Finland
}

(Submitted to Nucl. Phys. A on March 23, 2001)

The centrality dependence of the charged multiplicity, transverse energy, and elliptic flow coefficient is studied in a hydrodynamic model, using a variety of different initializations which model the initial energy or entropy production process as a hard or soft process, respectively. While the charged multiplicity depends strongly on the chosen initialization, the $p_{\mathrm{T}}$-integrated elliptic flow for charged particles as a function of charged particle multiplicity and the $p_{\mathrm{T}^{-}}$ differential elliptic flow for charged particles in minimum bias events turn out to be almost independent of the initial energy density profile.

PACS numbers: 25.75-q, 25.75.Ld

Keywords: Relativistic heavy-ion collisions; Elliptic flow; Hydrodynamic model

\section{INTRODUCTION}

Elliptic flow [1] is a collective flow pattern which develops in non-central relativistic heavy-ion collisions as a result of the spatial deformation of the initial transverse overlap area. It requires rescattering among the produced particles as a mechanism to map the initial spatial deformation of the reaction zone onto the finally observed hadron momentum distributions. It is quantified by the second harmonic coefficient $v_{2}$ of an azimuthal Fourier decomposition of the measured spectrum $d N /\left(d y p_{\mathrm{T}} d p_{\mathrm{T}} d \phi\right)$ 2. Its magnitude $v_{2}$ and its shape $v_{2}\left(p_{\mathrm{T}}\right)$ as a function of the hadron transverse momentum are sensitive to the scattering rate among the produced secondaries, especially during the dense early stage of the expansion [3]4. The largest elliptic flow signal, especially at high $p_{\mathrm{T}}$, arises in hydrodynamic simulations [1, 5[6] which assume local thermal equilibrium at every space-time point, i.e. essentially instantaneous thermalization or infinite scattering rate. Surprisingly, such hydrodynamic simulations [5] are in very good agreement with first results from $\sqrt{s}=130 \mathrm{AGeV} \mathrm{Au}+\mathrm{Au}$ collisions at the Relativistic Heavy Ion Collider (RHIC) [7 [11], up to transverse momenta of $1.5-2 \mathrm{GeV} / c$.

It was recently suggested [8,9, 12] that a combined analysis of the full set of hadronic single particle spectra and their elliptic flow as a function of collision centrality should allow to outline the domain of applicability of the hydrodynamic approach. Inside this domain such an analysis would constrain the initial baryon and energy density and the final freeze-out temperature sufficiently well to become sensitive to details of the equation of state (EOS) of the fireball matter [9]. In this context $v_{2}$ provides access to the EOS during the early expansion stage [3 5] which, at RHIC energies, is presumably in the quark-gluon plasma (QGP) phase. Direct verification of the phase transition between QGP at high energy density and a hadron resonance gas at lower energy density should then become possible by accurately measuring the excitation function of radial and elliptic flow [5].

In the hydrodynamic limit the EOS affects the elliptic flow signal through the velocity of sound, $c_{s}=\sqrt{d P / d e}$ [1]. However, the sensitivity of $v_{2}$ on $c_{s}$ is not very strong, and even a first order phase transition, where $c_{s}$ vanishes in the mixed phase, affects $v_{2}$ only on the $20 \%$ level [5]. This makes accurate measurements and systematic theoretical checks indispensable. One possible source of ambiguity, which has not been systematically investigated in previous studies [1],5, 6, 8, 9, 12, is the sensitivity of the radial and elliptic flow pattern on the shape of the initial transverse density profile. The latter depends on the scaling of secondary particle production with the number of colliding nucleons which itself is controlled by the collision centrality. It was recently found [13] that the momentum-space anisotropy at high $p_{\mathrm{T}}$, where it is not of hydrodynamic origin but due to quark energy loss [14], is quite sensitive to the initial density profile. A good hydrodynamic baseline for the dependence of $v_{2}\left(p_{\mathrm{T}}\right)$ on the initial profiles may thus help to clarify the physics controlling the breakdown of the hydrodynamic model and the transition to the hard physics domain at high $p_{\mathrm{T}}$.

In the present work, we investigate five options which are expected to span the realistic range of possibilities. In the first four, either the initial energy or the initial entropy density are assumed to scale with either the number of wounded nucleons ("soft" or "non-perturbative" particle production) or the number of binary nucleonnucleon collisions ("hard" or "perturbative" particle production). In the fifth model perturbative particle production is modified by implementing gluon shadowing [15 in the initial state and by limiting the growth of the production cross section by gluon saturation in the final state ("saturation model" [16,17]). This brings in 
some non-perturbative elements as well. In the first four parametrizations we normalize the initial energy density profile such that for central collisions we reproduce within errors the total charged multiplicity density at midrapidity, $d N_{\mathrm{ch}} /\left.d \eta\right|_{|\eta|<1}=555 \pm 10 \%$, as measured by PHOBOS for $\mathrm{Au}+\mathrm{Au}$ at $\sqrt{s}=130 \mathrm{AGeV}$ [18]. On the other hand, in the saturation model [16,17] the initial energy density is fixed by assuming that the transverse energy of produced minijets is entirely converted to thermalized energy density. The different scaling laws implied by the models then translate into different centrality dependencies of $d N_{\mathrm{ch}} / d \eta$ which can be tested against the new data presented recently 19,20.

The hydrodynamic model describes an adiabatic evolution from one local equilibrium state to another. Our version of the model assumes longitudinal boost invariance which implies conservation of the entropy rapidity density $d S / d y$. Using the relation between entropy and particle multiplicity in a thermalized system, the measured centrality dependence of the final multiplicity density $d N / d y$ can thus be mapped onto the centrality dependence of the initial parton multiplicity density at the point of thermalization. This allows to constrain models for the initial production of secondary particles, under the assumption of subsequent adiabatic evolution.

We show that different models for the initial energy and entropy production lead to different radial and elliptic flow patterns. At fixed impact parameter, these result in different $p_{\mathrm{T}}$-dependences of the spectra and of the differential elliptic flow $v_{2}\left(p_{\mathrm{T}}\right)$. However, the $p_{\mathrm{T}}$-integrated elliptic flow $v_{2}$ as a function of the final charged multiplicity density $d N_{\mathrm{ch}} / d y$ and the differential elliptic flow $v_{2}\left(p_{\mathrm{T}}\right)$ for minimum bias events show surprisingly little sensitivity to the model used for initializing the hydrodynamic evolution. These two observables have been used to argue for the applicability of the hydrodynamic model at RHIC energies [7], and they were shown to be sensitive to the EOS of the expanding matter [8.9]. Their insensitivity to the shape of the initial transverse energy density profile may prove to be crucial for the process of extracting reliable information on the EOS from a detailed analysis of the measured collective flow patterns.

\section{MODELS FOR THE INITIAL TRANSVERSE ENERGY DENSITY PROFILE}

\section{A. Soft particle production}

In $\mathrm{Pb}+\mathrm{Pb}$ collisions at the $\mathrm{SPS}$, the rapidity densities at midrapidity of both the total produced transverse energy $d E_{\mathrm{T}} / d y$, and of the charged multiplicity,

\footnotetext{
${ }^{1}$ We use $E_{\mathrm{T}}=\sum_{i} E_{i} \frac{p_{\mathrm{T}, i}}{\left|\boldsymbol{p}_{i}\right|}$ where the sum is over all particles.
}

$d N_{\mathrm{ch}} / d y$, scale approximately linearly with the number of participating nucleons, $N_{\text {part }}$ [2], except for very peripheral collisions with $N_{\text {part }} \lesssim 100$. Similar earlier observations in smaller collision systems and/or at lower energies have led to the notion that particle and transverse energy production can be described by the "Wounded Nucleon Model" [22] in which each participating nucleon contributes to the multiplicity and transverse energy only once in its first collision and not every time it suffers further collisions with other projectile or target nucleons. The validity of this model requires destructive interference effects between subsequent nucleon-nucleon collisions which are thought to be characteristic of nonperturbative or "soft" particle production processes. For two nuclei colliding at impact parameter $\boldsymbol{b}=b \boldsymbol{e}_{x}$, the density of wounded nucleons in the transverse plane (parametrized by $s=(x, y)$ ) can be calculated from the simple geometric formula (Glauber ansatz, see [23,24 and references therein):

$$
\begin{aligned}
n_{\mathrm{WN}}(\boldsymbol{s} ; \boldsymbol{b}) & =T_{A}\left(\boldsymbol{s}+\frac{1}{2} \boldsymbol{b}\right)\left[1-\left(1-\frac{\sigma T_{B}\left(\boldsymbol{s}-\frac{1}{2} \boldsymbol{b}\right)}{B}\right)^{B}\right] \\
& +T_{B}\left(\boldsymbol{s}-\frac{1}{2} \boldsymbol{b}\right)\left[1-\left(1-\frac{\sigma T_{A}\left(\boldsymbol{s}+\frac{1}{2} \boldsymbol{b}\right)}{A}\right)^{A}\right] .
\end{aligned}
$$

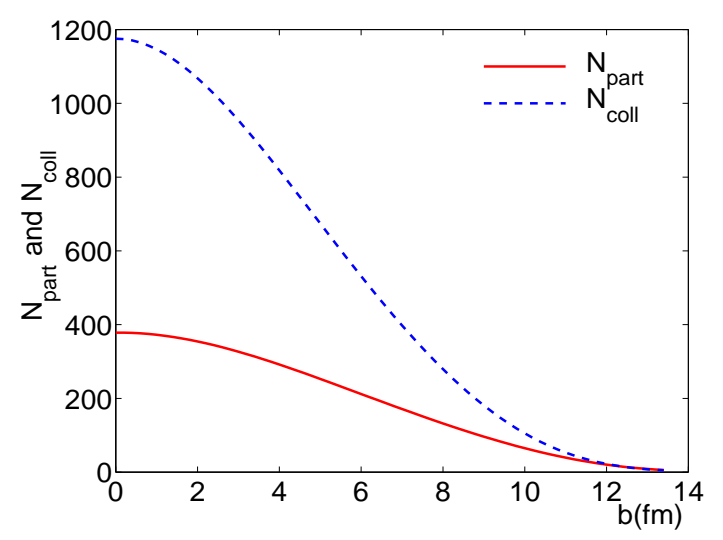

FIG. 1. Number of participating ("wounded") nucleons and of binary nucleon-nucleon collisions as functions of impact parameter. This and all following figures refer to $\mathrm{Au}+\mathrm{Au}$ collisions at $\sqrt{s}=130 \mathrm{~A} \mathrm{GeV}$.

$T_{A}$ is the nuclear thickness function of nucleus $A$,

$$
T_{A}(s)=\int_{-\infty}^{+\infty} d z \rho_{A}(s, z),
$$

with the density $\rho_{A}$ given by a Woods-Saxon profile,

$$
\rho_{A}(\boldsymbol{r})=\frac{\rho_{0}}{1+\exp \left[\left(r-R_{0}\right) / \xi\right]},
$$

and similarly for nucleus $B$. For the Woods-Saxon profile we take standard parameter values, $R_{0}=1.12 A^{1 / 3}-$ $0.86 A^{-1 / 3} \mathrm{fm}$ for the radius and $\xi=0.54 \mathrm{fm}$ for the surface diffuseness. The integral over (1) gives the number 
of "wounded" or "participating" nucleons, $N_{\text {part }} \equiv N_{\mathrm{WN}}$, as a function of impact parameter (see Fig. 11). For the total nucleon-nucleon cross section at $\sqrt{s}=130 \mathrm{~A} \mathrm{GeV}$ we take $\sigma=40 \mathrm{mb}$.

\section{Model $\mathrm{sWN}$}

Hydrodynamics with boost-invariant longitudinal expansion conserves the entropy per unit rapidity $d S / d y$. At fixed freeze-out temperature and chemical potential, $d S / d y$ is related one-to-one to the measured charged multiplicity density, $d N_{\mathrm{ch}} / d y$. If the latter scales linearly with the number of wounded nucleons, $N_{\mathrm{WN}}(b)=\int d^{2} s n_{\mathrm{WN}}(\boldsymbol{s} ; \boldsymbol{b})$, barring a strong $b$-dependence of the freeze-out conditions it is natural to assume that the initial entropy density in the transverse plane is proportional to that of the wounded nucleons [1,25]:

$$
s\left(\boldsymbol{s} ; \tau_{0} ; \boldsymbol{b}\right)=K_{s}\left(\tau_{0}\right) n_{\mathrm{WN}}(\boldsymbol{s} ; \boldsymbol{b}) .
$$

At SPS and RHIC energies the net baryon density is so small that its influence on the pressure and thus on the developing flow pattern is hardly noticeable. For simplicity we can thus also parametrize the initial net baryon density $n$ as being proportional to $n_{\mathrm{WN}}$ :

$$
n\left(\boldsymbol{s} ; \tau_{0} ; \boldsymbol{b}\right)=K_{n}\left(\tau_{0}\right) n_{\mathrm{WN}}(\boldsymbol{s} ; \boldsymbol{b}) .
$$

Equations (4) and (5) together define the first model for the initialization, $\mathrm{sWN}$, to be studied below.

The implementation of the initialization (14) into the hydrodynamic computer code is complicated by the fact that in our formulation the hydrodynamic equations propagate the fields $n(x)$ and $e(x)$ (and not $s(x)$ ). The initialization (बi thus requires the additional step of computing $e\left(\boldsymbol{s} ; \tau_{0} ; \boldsymbol{b}\right)$ from $s\left(\boldsymbol{s} ; \tau_{0} ; \boldsymbol{b}\right)$ by solving an implicit equation resulting from the thermodynamic identity

$$
T(e, n) s=e+p(e, n)-\mu(e, n) n,
$$

where $T(e, n), p(e, n)$, and $\mu(e, n)$ are tabulated values for the temperature, pressure and baryon chemical potential corresponding to the selected equation of state.

\section{Model eWN}

In a hydrodynamic approach with fixed freeze-out conditions, it is strictly speaking not possible that both the transverse energy $d E_{\mathrm{T}} / d y$ and the charged multiplicity $d N_{\mathrm{ch}} / d y$ scale linearly with $N_{\mathrm{WN}}$. During the hydrodynamic evolution thermal energy is converted into longitudinal and transverse flow energy, and the time available for this conversion increases with the number of wounded nucleons. Since the transverse flow increases the average transverse energy per particle, $d E_{\mathrm{T}} / d y$ should rise more quickly with $N_{\mathrm{WN}}$ than $d N_{\mathrm{ch}} / d y$. There is some indication for this to happen in the WA98 data from $\mathrm{Pb}+\mathrm{Pb}$ collisions at the SPS 21], where $E_{\mathrm{T}} / N_{\mathrm{ch}}$ at midrapidity rises slightly from very peripheral to semiperipheral collisions and then saturates from semiperipheral to central collisions. However, the experimental effect is small and not clearly statistically significant.

Given this unclear experimental situation, and because it simplifies the initialization process, we assumed in our previous work [5, 6,8,9. that it is the initial energy density (and not the entropy density) which scales with the density of wounded nucleons in the transverse plane:

$$
e\left(\boldsymbol{s} ; \tau_{0} ; \boldsymbol{b}\right)=K_{e}\left(\tau_{0}\right) n_{\mathrm{WN}}(\boldsymbol{s} ; \boldsymbol{b}) .
$$

Equation (7) together with Eq. (5) defines our second initialization model, eWN. The effect of flow on $E_{\mathrm{T}} / N_{\mathrm{ch}}$ at RHIC and its dependence on the initialization of the hydrodynamic evolution will be discussed in Section III.

\section{B. Hard particle production}

At higher and higher collision energies, one expects that hard collisions among quarks and gluons from the nuclear structure functions become more and more important and eventually dominate secondary particle production 26]. In this limit secondary particle production is a result of incoherent parton-parton collisions, and each nucleon-nucleon collision contributes equally to the cross section. Particle and energy production should then be related to the distribution of the number of binary nucleon-nucleon collisions in the transverse plane. It is given in terms of the nuclear thickness functions (2) by

$$
\frac{d N_{A B}^{\text {coll }}(\boldsymbol{s} ; \boldsymbol{b})}{d^{2} s}=\sigma T_{A}\left(\boldsymbol{s}+\frac{1}{2} \boldsymbol{b}\right) T_{B}\left(\boldsymbol{s}-\frac{1}{2} \boldsymbol{b}\right),
$$

where $\sigma$ is the nucleon-nucleon cross section. The integral of (8) is the nuclear overlap function, $T_{A B}(\boldsymbol{b})=$ $\int d^{2} s T_{A}\left(\boldsymbol{s}+\frac{1}{2} \boldsymbol{b}\right) T_{B}\left(s-\frac{1}{2} \boldsymbol{b}\right)$, which is normalized to $A B$ : $\int d^{2} b T_{A B}(\boldsymbol{b})=A B$. It gives the total number of binary collisions as a function of impact parameter, $N_{\text {coll }}(b)$, which is also shown in Fig. 田.

\section{Model $\mathrm{sBC}$}

If the system of secondary particles thermalizes quickly via elastic collisions, their number density defines, up to a proportionality constant, the initial entropy density at the beginning of the hydrodynamic expansion:

$$
s\left(\boldsymbol{s} ; \tau_{0} ; \boldsymbol{b}\right)=\tilde{K}_{s}\left(\tau_{0}\right) T_{A}\left(\boldsymbol{s}+\frac{1}{2} \boldsymbol{b}\right) T_{B}\left(\boldsymbol{s}-\frac{1}{2} \boldsymbol{b}\right) .
$$

Assuming that the initial net baryon density in the transverse plane can also be calculated perturbatively from the nuclear structure functions [27, we write 


$$
n\left(\boldsymbol{s} ; \tau_{0} ; \boldsymbol{b}\right)=\tilde{K}_{n}\left(\tau_{0}\right) T_{A}\left(\boldsymbol{s}+\frac{1}{2} \boldsymbol{b}\right) T_{B}\left(\boldsymbol{s}-\frac{1}{2} \boldsymbol{b}\right) .
$$

As discussed in Section II A 1, the results do not depend on whether we use (10) or (5). The combination of Eqs. (9) and (10) defines model sBC for the initialization.

\section{Model eBC}

Within the perturbative approach to particle production one can also argue that each nucleon-nucleon collision contributes with equal probability not only to the number of produced secondaries, but also to the energy carried by them. This leads to the ansatz that the initial energy (and not the entropy) density is proportional to the density of binary collisions in the transverse plane:

$$
e\left(\boldsymbol{s} ; \tau_{0} ; \boldsymbol{b}\right)=\tilde{K}_{e}\left(\tau_{0}\right) T_{A}\left(\boldsymbol{s}+\frac{1}{2} \boldsymbol{b}\right) T_{B}\left(\boldsymbol{s}-\frac{1}{2} \boldsymbol{b}\right) .
$$

This equation together with Eq. (10) defines model eBC for the initialization.

\section{The saturation model}

In the saturation model [16,17] the production of partons becomes inhibited below a saturation scale $p_{\text {sat }}$, determined as the transverse momentum scale where the produced partons start to overlap transversally. The formation time of the QGP at each point $s$ is given by $\tau_{\text {sat }}=1 / p_{\text {sat }}(\sqrt{s}, A, B, \boldsymbol{s} ; \boldsymbol{b})$. The local initial energy density profile can then be worked out at the central slice as in 28],

$$
\begin{aligned}
e(\boldsymbol{s} ; \boldsymbol{b})= & \frac{d E_{\mathrm{T}}^{\mathrm{pQCD}}}{d^{2} s d z} \\
= & T_{A}\left(s+\frac{1}{2} \boldsymbol{b}\right) T_{B}\left(\boldsymbol{s}-\frac{1}{2} \boldsymbol{b}\right) \\
& \times \sigma\left\langle E_{\mathrm{T}}\right\rangle\left(\sqrt{s}, p_{\mathrm{sat}}, \Delta y, A, B, \boldsymbol{s} ; \boldsymbol{b}\right) \cdot \frac{1}{\tau_{\mathrm{sat}} \Delta y},
\end{aligned}
$$

where $d z \approx \tau_{\text {sat }} \Delta y$ within the central rapidity unit $\Delta y$. The first $E_{\mathrm{T}}$-moment of the minijet distribution, $\sigma\left\langle E_{\mathrm{T}}\right\rangle$ 26,27, is computed in lowest order pQCD as the first $p_{\mathrm{T}^{-}}$ moment of the distributions of minijets with $p_{\mathrm{T}} \geq p_{\mathrm{sat}}$ and $y$ within $\Delta y$. The EKS98 shadowing [15] of the parton distributions is included and the NLO contributions to $\sigma\left\langle E_{\mathrm{T}}\right\rangle$ 29] are simulated by an approximate factor $K=2$, as also done in [16, 17].

In 17] a fully saturated system was considered by extending the computation down to very low values of $p_{\text {sat }}=0.5 \mathrm{GeV}$, and neglecting the tails of the number (energy) density distributions at large transverse distances. Now, for the hydrodynamic description, we have to consider the tails also. To maintain the spirit of the local saturation model of [17], we restrict the saturation to the regions where $p_{\text {sat }} \geq 0.75 \mathrm{GeV}$ and compute the tail of the energy density profile from Eq. (12) with $p_{\text {sat }}=0.75 \mathrm{GeV}$. Through this procedure, the multiplicity of 17] in central collisions is recovered (if computed as in 171$)$. For central collisions, the tail contributes only $7 \%$ to the total multiplicity. We would like to emphasize that we have not tried to fit the saturation model to the centrality data but, rather, to keep the approach as close to the orginal local saturation idea [17] as possible.

As discussed in [16], from the energy per particle point of view the system looks thermal already at saturation. The same can be shown to hold also in the locally saturated system [17]. Within the saturation framework it is therefore meaningful to switch on the hydrodynamic evolution already at $\tau_{\text {sat }}$ which now is a local quantity.

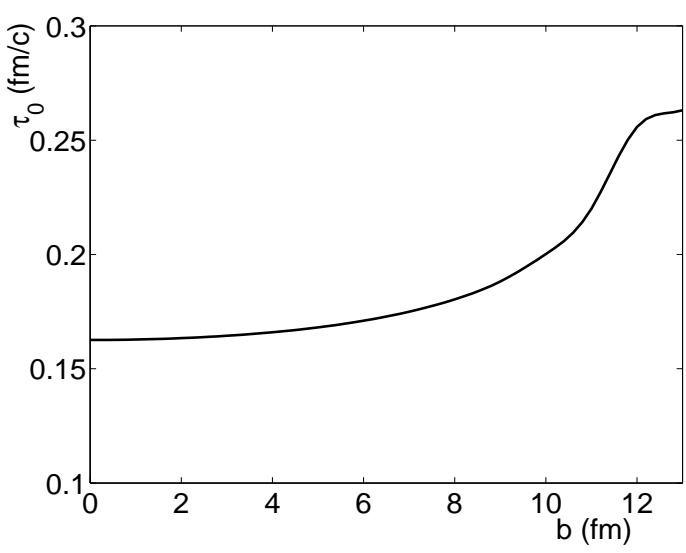

FIG. 2. The formation and initial thermalization time $\tau_{\text {sat }}(b) \equiv \tau_{\text {sat }}(s=0 ; \boldsymbol{b})=1 / p_{\text {sat }}$ in the saturation model, as a function of impact parameter $b$.

For the hydrodynamic set-up used in this study, however, the density profiles at a constant initial time are required. To circumvent this problem, we consider two possibilities: first, for each impact parameter $\boldsymbol{b}$, we simply use the earliest time, i.e. $\tau(\boldsymbol{s}=0)$, as the initial time. These are shown in Fig. 2. Notice that the maximum initial time is $(0.75 \mathrm{GeV})^{-1}=0.26 \mathrm{fm} / c$, and that a constant initial time is a good approximation for the central region and for small impact parameters. Alternatively, the energy densities computed from Eq. (12) can be evolved at each $\boldsymbol{s}$ and at each $\tau_{\text {sat }}(\boldsymbol{s} ; \boldsymbol{b})$ to $\tau_{i}=0.26 \mathrm{fm} / c$ assuming boost-invariant flow without transverse expansion:[] $e\left(\boldsymbol{s} ; \tau_{i} ; \boldsymbol{b}\right)=e\left(\boldsymbol{s} ; \tau_{\text {sat }}(\boldsymbol{s} ; \boldsymbol{b}) ; \boldsymbol{b}\right) \times\left(\tau_{\mathrm{sat}}(\boldsymbol{s} ; \boldsymbol{b}) / \tau_{i}\right)^{4 / 3}$. We have checked, however, that the latter procedure leads only to a few percent increase in the central multiplicities relative to the former one. Therefore, we take in the following the initial time for the saturation model from Fig. 2 and use $e\left(\boldsymbol{s} ; \tau_{\text {sat }}(b) ; \boldsymbol{b}\right)$ as the initial profile

\footnotetext{
${ }^{2}$ We thank P.V. Ruuskanen for helpful discussions related to this point.
} 
for the transversally expanding hydrodynamics, as discussed next.

\section{Initial energy density and spatial anisotropy}

Figure 3 shows the initial energy density profiles for the different initialization models. $(x$ is the direction of the impact parameter $\boldsymbol{b}$, while $y$ points perpendicular to the reaction plane.) For models sWN, eWN, sBC and $\mathrm{eBC}$ the profiles are normalized such that in each case the total charged multiplicity per unit pseudorapidity at $b=0$ is $d N_{\mathrm{ch}} / d y=670$ at $y=0$. For model eWN \& this corresponds to $d N_{\mathrm{ch}} /\left.d \eta\right|_{|\eta|<1}=545$ for the $6 \%$ most central collisions (a bit less for the three other models). Within errors, this is consistent with the first published PHOBOS data [18], but slightly below the more accurate recent data from PHENIX and PHOBOS [19,20]. To preserve consistency with our previous publications [8],9], we decided against increasing our normalization of $d N_{\mathrm{ch}} / d \eta$ to the new data since this would have implied retuning the initial conditions and freeze-out temperature in order to keep the spectra and elliptic flow unchanged.
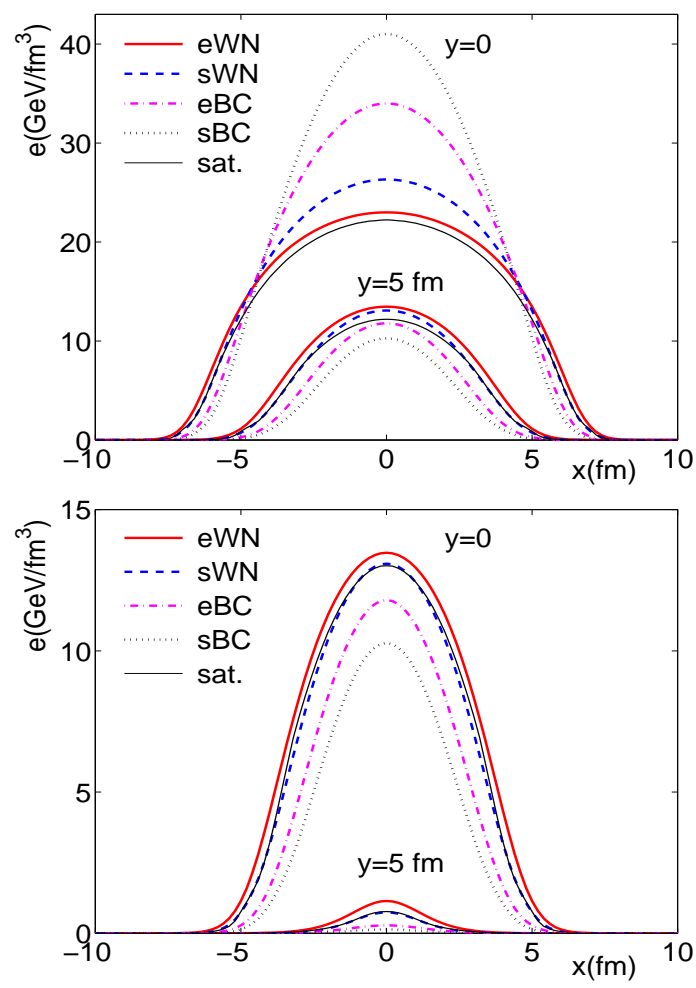

FIG. 3. Initial energy density profiles in the transverse plane for the five different initialization models described in this Section. Top: $b=0$. Bottom: $b=10 \mathrm{fm}$. In each case two cuts in $x$-direction are shown, one for $y=0$ and one for $y=5 \mathrm{fm}$. For the saturation model ("sat.") the profile was hydrodynamically propagated from the initial thermalization time assumed in that model to $\tau_{0}=0.6 \mathrm{fm} / c$ where the hydrodynamic evolution was started for the other four models.
For the comparison with the other models in Fig. 3, the profile obtained from the saturation model was evolved from the formation time $\tau_{\text {sat }}(b)$ (see Fig. 2) to $\tau_{0}=0.6 \mathrm{fm} / c$, the time at which the hydrodynamic evolution was started for the other four initializations. Within the line widths of Fig. 3, it did not matter whether this scaling was done by assuming only boost-invariant longitudinal expansion, $e\left(s, \tau_{0}\right)=e\left(s, \tau_{\text {sat }}\right) \times\left(\tau_{\text {sat }} / \tau_{0}\right)^{4 / 3}$, or by including also the transverse expansion.

Since for a thermalized parton gas at nearly vanishing net baryon density $e \sim s^{4 / 3}$, Eq. (9) gives distributions which are more sharply peaked at the origin than Eq. (11). We will see that this results in a different centrality dependence of the total entropy per unit rapidity $d S / d y$ (which, for boost-invariant longitudinal expansion, is proportional to the integral of $s(\boldsymbol{s})$ over the transverse plane). Model eBC interpolates between model sWN, where $d S / d y \sim N_{\text {part }}$, and model sBC, where $d S / d y \sim N_{\text {coll }} \sim N_{\text {part }}^{4 / 3}$ (the latter proportionality was checked numerically to hold with excellent accuracy over the entire impact parameter range). This is similar to the HIJING model [30] where the final charged particle rapidity density is a linear superposition of a soft component, scaling with $N_{\text {part }}$, and a hard component which scales with $N_{\text {coll }} \sim N_{\text {part }}^{4 / 3}$. In contrast to the models here, however, HIJING has no rescattering among the produced particles and thus no collective flow. Finally, the saturation model is seen to be close to model eWN for central collisions, while for semiperipheral collisions it nearly coincides with model sWN.

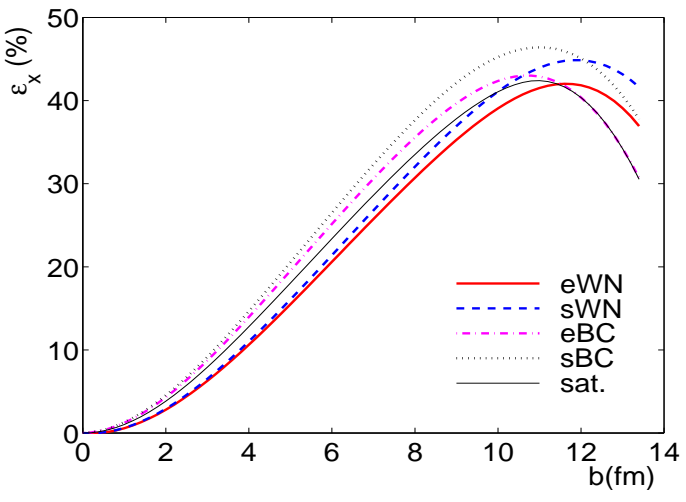

FIG. 4. Initial spatial anisotropy as a function of impact parameter, for the different initializations.

Figure 4 shows the initial spatial anisotropy

$$
\epsilon_{x}(b) \equiv \delta(b)=\frac{\left\langle\left\langle y^{2}-x^{2}\right\rangle\right\rangle}{\left\langle\left\langle y^{2}+x^{2}\right\rangle\right\rangle}
$$

as a function of impact parameter, evaluated with the initial transverse energy density as weight function, for the five initialization models studied here. At fixed impact parameter, $\epsilon_{x}$ varies by up to $20 \%$. Since the hydrodynamic evolution maps the initial spatial anisotropy onto 


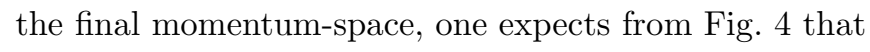
the impact parameter dependence of the elliptic flow $v_{2}$ should show a similar sensitivity to the initialization. We will see that, except for the saturation model, this model sensitivity is almost exactly cancelled by the corresponding variations in the impact parameter dependence of the produced charged particle multiplicity (i.e. entropy).

\section{CENTRALITY DEPENDENCE OF MULTIPLICITY AND TRANSVERSE ENERGY}

Using the initial transverse energy and baryon density profiles from the previous section and the numerical code described in [6], we solve the hydrodynamic equations for the transverse evolution of the reaction zone, assuming boost-invariant expansion in the longitudinal direction. We study only $\mathrm{Au}+\mathrm{Au}$ collisions at $\sqrt{s}=130 \mathrm{AGeV}$. For the models eWN, sWN, eBC and $\mathrm{sBC}$ we use the same initial time $\tau_{0}=0.6 \mathrm{fm} / c$ as in [8,9] to start the hydrodynamic expansion. For the saturation model the hydrodynamic expansion is started at $\tau_{\text {sat }}(b)$ shown in Fig. 2. All calculations reported here are performed with EOS Q, an equation of state with a first order phase transition from a hadron resonance gas to a non-interacting quark-gluon gas at $T_{\mathrm{c}}=164 \mathrm{MeV}$ 31], and freeze-out at $T_{\mathrm{f}}=120 \mathrm{MeV}$. The sensitivity of the spectra and elliptic flow to the EOS and freeze-out temperature were studied in 8,96.

\section{A. Charged particle multiplicity}

Figure 5 shows the final charged particle rapidity density per participating nucleon pair resulting from the different initializations. In the bottom panel the rapidity density at midrapidity is converted to pseudorapidity density by the transformation

$$
\frac{d N_{\mathrm{ch}}}{d \eta}=\sum_{i \in \text { charged }} \int p_{\mathrm{T}} d p_{\mathrm{T}} \frac{p_{\mathrm{T}}}{\sqrt{m_{i}^{2}+\boldsymbol{p}^{2}}} \frac{d N_{i}}{d y p_{\mathrm{T}} d p_{\mathrm{T}}}
$$

setting $y=p_{\mathrm{L}}=0$. At fixed freeze-out temperature $d N_{\mathrm{ch}} / d y$ is a direct measure for the entropy density $d S / d y$, and the approximate constancy of the curve for model sWN thus reflects the approximate conservation of entropy by the hydrodynamic evolution. In fact, the slight increase of $\left(d N_{\mathrm{ch}} / d y\right) / N_{\text {part }}$ for small values of $N_{\text {part }}$ (Fig. 5 , upper panel) can be traced back to a small amount of entropy production by deflagration shocks which arise during the hydrodynamic expansion stage as a consequence of the first order phase transition [32] once the initial energy density in the center of the reaction zone increases above the critical value for QGP formation.

Model eWN (solid line in Fig. 5) was used in Refs. [0.6, 8.9, while model sWN was employed in [1, 12, 25.
Fig. 5 shows that both initialization models are disfavoured by the data, model eWN more so than model sWN. The saturation model produces a rather similar centrality dependence of the charged multiplicity as model eWN.
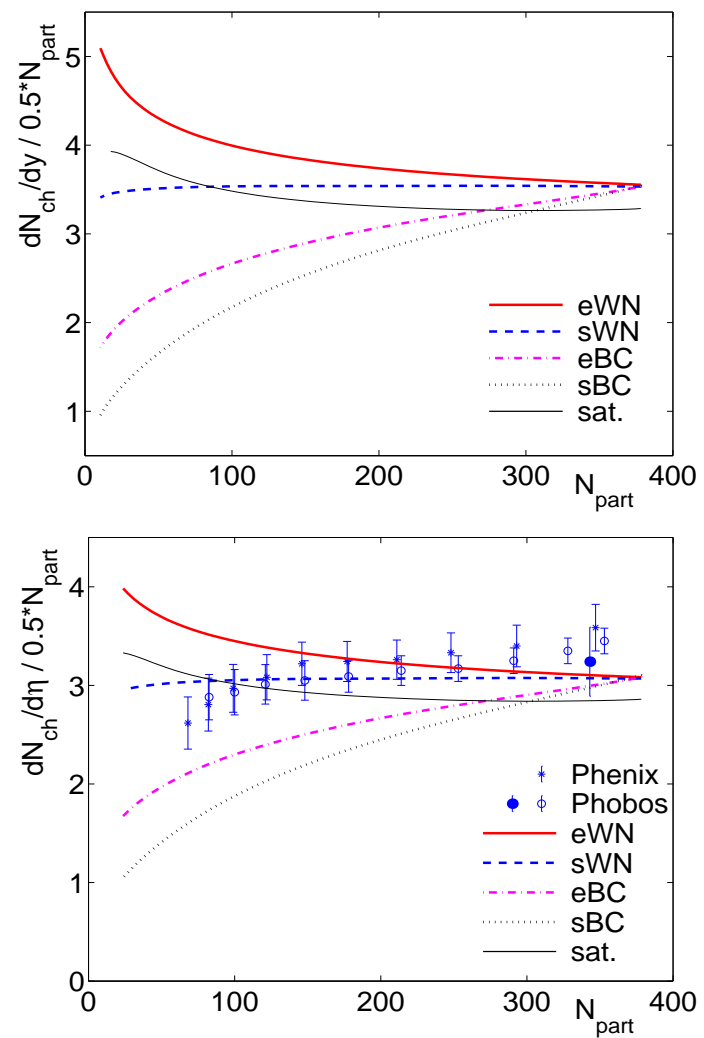

FIG. 5. Charged particle yield per participating nucleon pair at midrapidity as a function of the number of participants. All curves were normalized to $d N_{\mathrm{ch}} / d y=670$ for central $(b=0)$ collisions (see discussion below Fig. 3). The top panel gives the rapidity, the bottom panel the pseudorapidity density. The data are taken from Refs. 1820.

As expected, the saturation model results are close to those obtained in Ref. [17]. The careful reader notices, however, that the values $\left(d N_{\mathrm{ch}} / d y\right) /\left(0.5 N_{\text {part }}\right)$ for the saturation model shown in Fig. 同are $\mathcal{O}(10 \%)$ lower than those shown in Fig. 4 of [17]. This is due to a number of partially cancelling effects [28]: (i) Inclusion of the quark degrees of freedom increases the initial entropy by about $20 \%$ compared to 17]. (ii) In a realistic hadron resonance gas containing also heavy particles, at a freeze-out temperature of $T_{\mathrm{f}}=120 \mathrm{MeV}$ each particle carries on average 4.87 units of entropy, instead of the 4 units assumed in [17] (which is a good approximation in the ultrarelativistic limit). This decreases the total multiplicity from that in [17]. (iii) Finally, the decay of unstable resonances changes the fraction of charged particles from $2 / 3$ [17] to $\approx 0.62$ of the total multiplicity. The net reduction effect for the final charged multiplicity rapidity density is about $10 \%$ as mentioned. 
The best representation of the shape of the data, even down to rather peripheral collisions (low $N_{\text {part-values), }}$ seems to be given by model eBC, once appropriately renormalized to the new, slightly higher multiplicity densities in central collisions. Alternatively, one may try a linear combination of the sWM and sBC parametrizations, with a large sWN and a smaller sBC contribution 30,33 (the latter is apparently absent at the SPS where the multiplicity scales linearly with $\left.N_{\text {part }}\right)$. As discussed in Section IIC, the main difference between the model $\mathrm{eBC}$ and the saturation model is that the $\boldsymbol{s}$-dependence in eBC is contained only in the product $T_{A}\left(s+\frac{1}{2} \boldsymbol{b}\right) T_{B}\left(\boldsymbol{s}-\frac{1}{2} \boldsymbol{b}\right)$ whereas in the saturation model 17] also the cross sections (and their moments) depend on $s$ through the local saturation scale $p_{\text {sat }}(s)$. The data do not appear to support this particular implementation [17] of gluon saturation at the present RHIC energy of $\sqrt{s}=130 \mathrm{~A} \mathrm{GeV}$ for the non-central collisions. In central collisions, however, parton saturation is not excluded as the dominant mechanism. We conclude that obviously the saturation region is now extended too far in transverse direction and that the $\mathrm{eBC}$ tails in the initial energy density profile (which were not included at all in [17]) should be given more weight than they have now. On the other hand, the data strongly indicate that the initial energy deposition process does involve a component which scales with the number of binary nucleon-nucleon collisions. We refrain from a discussion whether or not this indeed proves the onset of "hard" perturbative physics at RHIC [30.

\section{B. Transverse energy per particle}

Hydrodynamic flow is a result of the conversion of a fraction of the thermal energy into collective flow kinetic energy, through work done by the thermodynamic pressure. As a consequence, the thermal energy per particle decreases as a function of time. If the reaction zone were to undergo boost-invariant expansion only along the longitudinal direction, $d S / d y \sim d N_{\mathrm{ch}} / d y$ would be constant and $d E_{\mathrm{T}} / d y$ would be proportional to the thermal energy density. The reduced thermal energy per particle would thus be directly reflected in the finally observed transverse energy per charged particle, $\left(d E_{\mathrm{T}} / d y\right) /\left(d N_{\mathrm{ch}} / d y\right)$ [34. Since in more central collisions the thermodynamic pressure does longitudinal hydrodynamic work for a longer time, the finally observed ratio $\left(d E_{\mathrm{T}} / d y\right) /\left(d N_{\mathrm{ch}} / d y\right)$ should increase much more slowly with $N_{\text {part }}$ than the initial energy per particle 35] established during the energy deposition process. In reality, the system undergoes not only longitudinal, but also transverse expansion. The transverse collective flow adds a kinetic contribution to $E_{\mathrm{T}}$ which reduces the diluting effect on $E_{\mathrm{T}} / N_{\mathrm{ch}}$ from the longitudinal expansion.
This shows that the centrality dependence of $E_{\mathrm{T}} / N_{\mathrm{ch}}$ is an interesting observable which reflects the interplay of the centrality dependences of initial transverse energy and particle production, longitudinal work done by the hydrodynamic pressure, and radial flow. In Figure 6 we show the corresponding results from the hydrodynamic model for $\mathrm{Au}+\mathrm{Au}$ collisions at $\mathrm{RHIC}(\sqrt{s}=130 \mathrm{AGeV})$ for the five different initialization models. For comparison we also show the centrality dependence for $\mathrm{Pb}+\mathrm{Pb}$ collisions at the SPS, for model eWN with parameters adjusted to the measured spectra 5:60. Comparing the eWN curves for SPS and RHIC, one sees that the larger radial flow at RHIC has very little effect on the average $E_{\mathrm{T}}$ per particle in the final state: the gain in transverse kinetic flow energy is largely compensated by more longitudinal work done at RHIC (smaller $\tau_{0}$, somewhat larger $\left.\tau_{\mathrm{f}}\right)$. The eWN curve at the SPS is consistent in shape with the WA98 data shown in Fig. 14 of [21], but at the upper edge of their systematic error band.

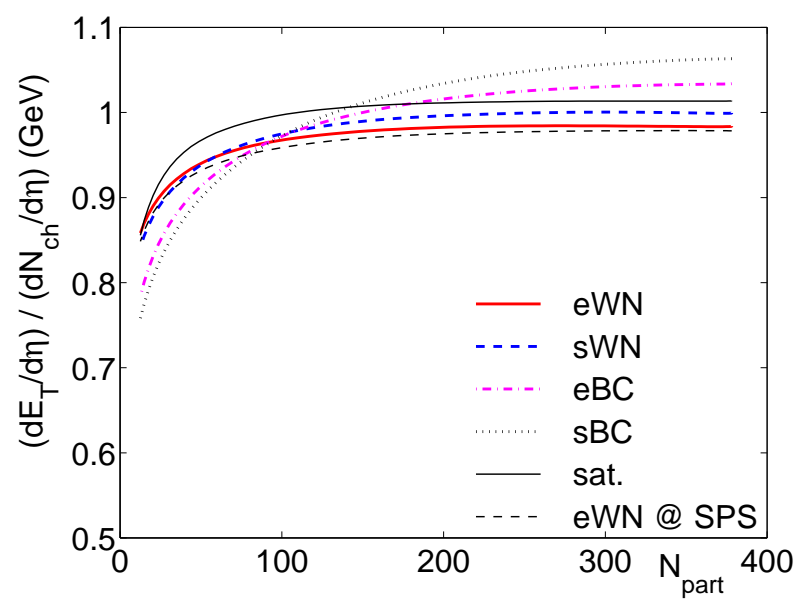

FIG. 6. Average transverse energy per charged particle at midrapidity as a function of the number of participants. Results are shown for all five initialization models for $\mathrm{Au}+\mathrm{Au}$ collisions at $\sqrt{s}=130 \mathrm{AGeV}$. In addition we plot the result from model eWN for $\mathrm{Pb}+\mathrm{Pb}$ collisions at $\sqrt{s}=17 A \mathrm{GeV}$, using the initial conditions from Ref. [5, 6] (thin dashed line).

At RHIC, the models eWN and sWN as well as the saturation model all give similarly flat centrality dependences for the average $E_{\mathrm{T}}$ per charged particle; they differ slightly in normalization and in how steeply this ratio drops for very peripheral collisions. The data in Figure 5 disfavour these initialization models. On the other hand, Model eBC, which works better in Figure 5, exhibits a markedly stronger centrality dependence of $E_{\mathrm{T}} / N_{\text {ch }}$, with a clearly visible slope below $N_{\text {part }} \simeq 250$. (This is even more true for the initialization sBC, but for that model the corresponding curve in Fig. 5 is too steep.) This reflects a similar strong centrality dependence of $\left(d E_{\mathrm{T}} / d y\right) /(d S / d y)$ in the initial state, which is only partially offset by the increasing amount of longitu- 
dinal hydrodynamic work done as the collisions become more central.

As recently pointed out in [35], a measurement of the ratio plotted in Fig. 6 will be very helpful in comple-

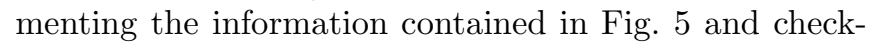
ing for the effects of longitudinal work. We note that non-equilibrium expansion scenarios like those studied in [35], where the matter performs less longitudinal work than predicted hydrodynamically, should lead to an even stronger dependence of $E_{\mathrm{T}} / N_{\mathrm{ch}}$ on $N_{\text {part }}$ than that shown in Fig. 6. In contrast, recent data from the PHENIX Collaboration 36 exhibit the opposite tendency: they are flatter than the eBC curve in Fig. 6 and, in fact, quantitatively consistent in magnitude and shape with the WA98 data at the SPS [21]. It remains to be seen whether e.g. a linear combination of a large sWN with a small sBC component (which can be made to agree with the multiplicity data in Fig. 5 [33]) leads to a sufficiently flat behaviour in Fig. 6 to be consistent with the PHENIX data [36], and whether the magnitude of $E_{\mathrm{T}} / N_{\mathrm{ch}}$ can be lowered by replacing the unrealistic chemical composition from the hydrodynamical model by a more realistic one (our hydrodynamic model assumes that chemical equilibrium is preserved until kinetic freeze-out at $T_{\mathrm{f}}=120 \mathrm{MeV}$ whereas preliminary STAR data indicate a much higher chemical freeze-out temperature of around $170-180 \mathrm{MeV}$ [11).

\section{RADIAL AND ELLIPTIC FLOW}

\section{A. Radial flow}

The single particle spectra for positive pions and antiprotons for the different initialization models are shown in Figure 8, for two representative values of the impact parameter. For central $(b=0)$ collisions, the systematics of the $m_{\mathrm{T}}$-slopes shows that for models eWN, sWN, eBC and $\mathrm{sBC}$ the amount of radial flow created in the collision increases in the same order as the central initial energy density shown in Figure 3. The saturation model is an exception: it gives larger radial flow than both eWN and $\mathrm{sWN}$ although, at $\tau_{0}=0.6 \mathrm{fm} / c$, its central energy density is even below that of model eWN. The reason is that for the saturation model the hydrodynamic evolution starts earlier and the build-up of transverse flow thus begins already at time $\tau_{\text {sat }}(b)$ (see Fig. 2).

The effect of the initialization on the single particle spectra is significant, and switching from model eWN used in [9] to model eBC, which according to Figure 5 provides a better fit to the centrality dependence of the charged multiplicity, considerably flattens the single particle spectra predicted in Ref. [9] for central collisions. This model sensitivity is weaker for semiperipheral collisions $(b=8 \mathrm{fm})$, again in agreement with Figure 3 which shows that in this range the initial energy density profiles are rather similar for all the models. If hydrodynamics still applies to even more peripheral collisions, models $\mathrm{eBC}$ and $\mathrm{sBC}$ would there predict steeper spectra than models eWN and sWN.

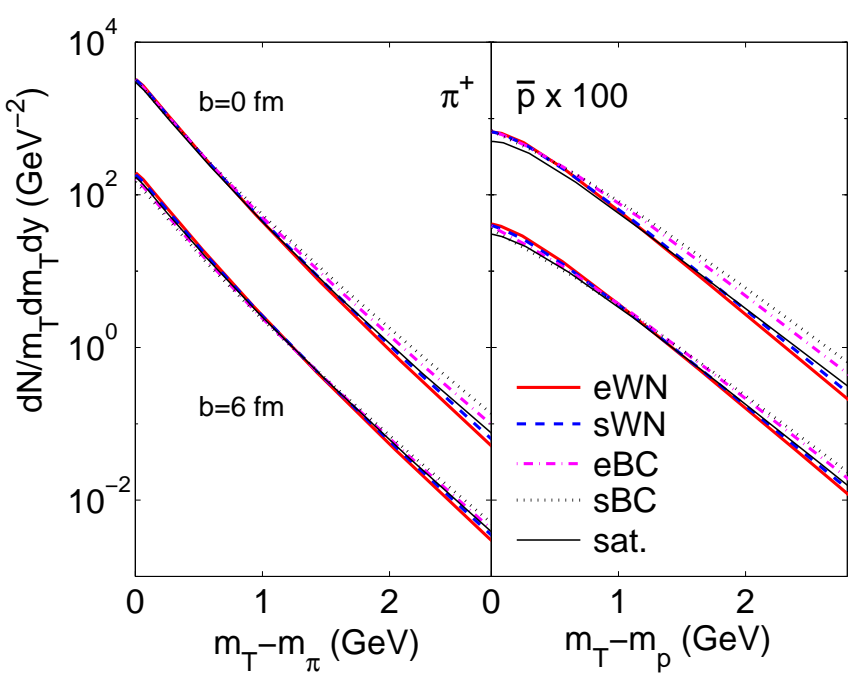

FIG. 7. Transverse mass spectra for positive pions and antiprotons, for central (upper set of curves) and semiperipheral collisions (lower set of curves, divided by 10 for clarity), for the five initialization models studied in this paper.

\section{B. Elliptic flow}

Figure 8 shows that for the four models eWN, sWN, $\mathrm{eBC}$, and $\mathrm{sBC}$ the differential elliptic flow $v_{2}\left(p_{\mathrm{T}}\right)$ follows a similar pattern as the radial flow: the model with the largest initial central energy density produces the strongest elliptic flow. The saturation model does not follow this systematics, which again is presumably due

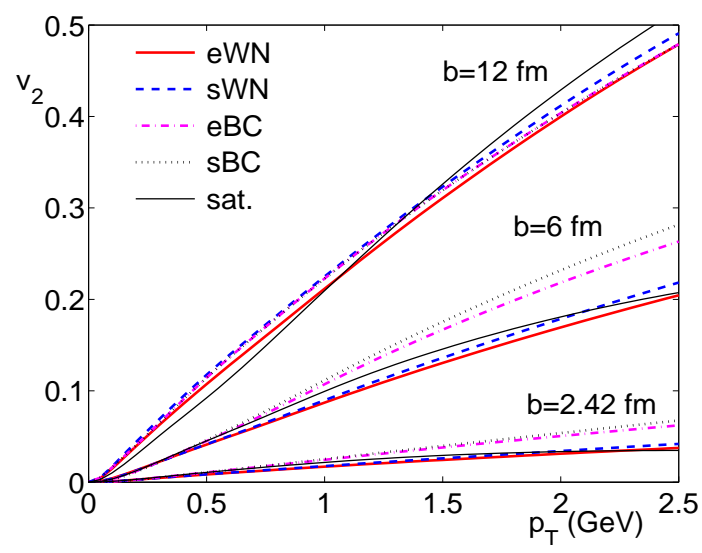

FIG. 8. The $p_{\mathrm{T}}$-differential elliptic flow $v_{2}\left(p_{\mathrm{T}}\right)$ for charged hadrons, at three representative impact parameters and for the five initialization models studied in this paper.

to the earlier start of the hydrodynamic evolution in this model. The impact parameter dependence of this 
model sensitivity is now controlled by the initial spatial anisotropy $\epsilon_{x}$. According to Figure 1 , for most of the impact parameter range it satisfies the hierarchy $\epsilon_{x}(\mathrm{eWN})<\epsilon_{x}(\mathrm{sWN})<\epsilon_{x}(\mathrm{sat})<\epsilon_{x}(\mathrm{eBC})<\epsilon_{x}(\mathrm{sBC})$, and this is clearly reflected in the slopes of $v_{2}\left(p_{\mathrm{T}}\right)$ for $p_{\mathrm{T}}<2 \mathrm{GeV} / c$, as shown in Figure 8. Again, the effect is significant and can reach up to $20 \%$. At large impact parameters, $b>10 \mathrm{fm}$, and for $p_{\mathrm{T}}>2 \mathrm{GeV} / c$ this simple ordering is broken.

In view of these results, it is somewhat of a pleasant surprise to see in Figure 9 that the $p_{\mathrm{T}}$-integrated elliptic flow $v_{2}$ for all charged hadrons, when plotted as a function of the midrapidity density of charged particle multiplicity as an experimentally easily accessible centrality measure, shows very little sensitivity to the initialization model. The models eWN, sWN, eBC, and sBC yield almost identical results, and only for the saturation model $v_{2}$ is slightly larger (except for the most peripheral collisions). Comparing the two extreme models eWN and sBC, one sees from Figure 5 that, at fixed charge multiplicity, the latter favours smaller impact parameters which correspond to smaller $\epsilon_{x}$ and $v_{2}\left(p_{\mathrm{T}}\right)$ (Figs. 1 and 7 , but flatter single particle spectra which give more weight to larger $v_{2}$ at higher $p_{\mathrm{T}}$ (Fig. 6). The net effect of this intricate interplay is an almost complete cancellation of these counteracting tendencies.

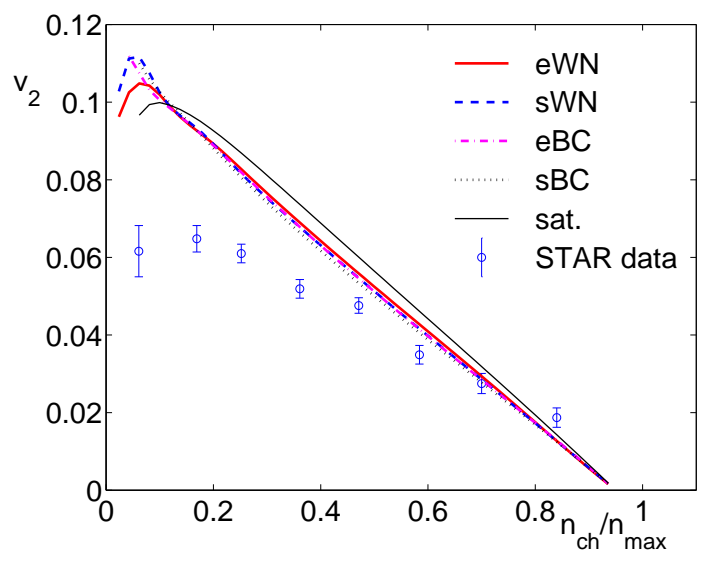

FIG. 9. The $p_{\mathrm{T}}$-integrated elliptic flow $v_{2}$ for charged hadrons as a function of midrapidity charged multiplicity density, for different initialization models. The data are taken from Ref. []]. (For a discussion of the horizontal axis $n_{\mathrm{ch}} / n_{\max }$ in theory and experiment, we refer to Refs. [7, 8].)

A similar cancellation happens for the differential anisotropy $v_{2}\left(p_{\mathrm{T}}\right)$ for charged hadrons in minimum bias events (left panel of Figure 10):

$$
v_{2}\left(p_{\mathrm{T}}\right)=\frac{\int b d b v_{2}\left(p_{\mathrm{T}} ; b\right) \frac{d N_{\mathrm{ch}}}{d y p_{\mathrm{T}} d p_{\mathrm{T}}}(b)}{\int b d b \frac{d N_{\mathrm{ch}}}{d y p_{\mathrm{T}} d p_{\mathrm{T}}}(b)} .
$$

At fixed $b$, the models with smaller elliptic flow are weighted with larger charged multiplicities, but slightly steeper spectra, and again the net result is an almost miraculous cancellation of all sensitivities to the initialization model for $p_{\mathrm{T}} \lesssim 1.5 \mathrm{GeV} / c$. Above $p_{\mathrm{T}}=1 \mathrm{GeV} / c$, the saturation model gives the largest $v_{2}\left(p_{\mathrm{T}}\right)$.

This cancellation does not carry over to heavier particles. The right panel in Figure 10 shows the differential elliptic flow $v_{2}\left(p_{\mathrm{T}}\right)$ for identified protons and antiprotons, which is seen to exhibit more significant variations as the initialization of the hydrodynamic model is changed. Generically, the model which gives the largest radial flow at small impact parameters produces the smallest proton elliptic flow at small values of $p_{\mathrm{T}}$. This is qualitatively consistent with the general analytic discussion of radial flow effects on the elliptic flow for heavy particles presented in Ref. [9]. Again, the saturation model gives the largest elliptic flow of all studied initializations for protons with $p_{\mathrm{T}}>1 \mathrm{GeV} / c$.

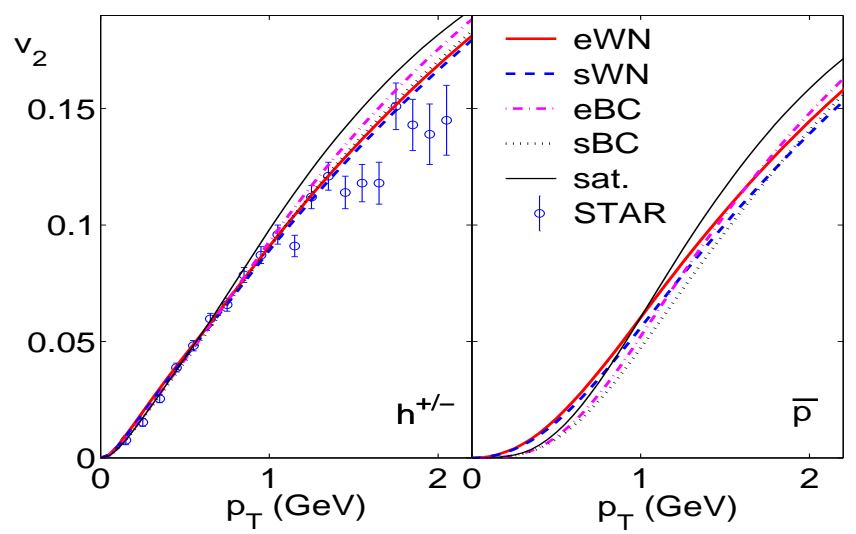

FIG. 10. The differential elliptic flow $v_{2}\left(p_{\mathrm{T}}\right)$ for minimum bias events, for all charged hadrons (left) and for identified protons or antiprotons (right). The curves correspond to different initialization models as indicated in the Figure. The data in the left panel are from Ref. (7).

\section{CONCLUSIONS}

Within a hydrodynamic model with exact longitudinal boost invariance, we have shown that the centrality dependence of the production of particles, transverse energy and transverse flow is significantly influenced by the shape of the initial energy density profile in the transverse plane. This profile is intimately related to the nature of the primary particle production process which converts beam energy into the matter forming the collision fireball. The available data for the charged multiplicity in $\mathrm{Au}+\mathrm{Au}$ collisions at RHIC as a function of collision centrality are compatible with an initial energy deposition process involving a component which scales with the number of binary nucleon-nucleon collisions. They strongly disfavour the wounded nucleon parametrization used in earlier hydrodynamic simulations [1,5, 6, 8, 9, 12,25]. In noncentral collisions they are, at the present RHIC energy, 
also at variance with predictions from the gluon saturation model [17. The best agreement with the multiplicity data at $\sqrt{s}=130 \mathrm{AGeV} 18$ 20 would be obtained by assuming that most of the initial thermalized energy density is proportional to the number of binary nucleonnucleon collisions, with a smaller "soft" contribution proportional to the number of wounded nucleons. If one instead parametrizes the initial entropy density as a superposition of "hard" and "soft" components, as implied by the approaches in 30,33, one needs a larger "soft" and smaller $(\sim 10 \%)$ "hard" component 33. A model with similar properties is obtainable also in the saturation approach considered here by limiting the saturation region to smaller transverse distances and thereby increasing the contribution from the tail which scales with the number of binary collisions. All these models predict an average transverse energy per particle in the final state which rises slowly with increasing number of participating nucleons. A measurement of $E_{\mathrm{T}} / N_{\text {ch }}$ as a function of $N_{\text {part }}$ is sensitive to the longitudinal work done during the hydrodynamic evolution and can be used to check the consistency of the hydrodynamic approach.

The centrality dependence of the $p_{\mathrm{T}^{-}}$-averaged elliptic flow $v_{2}$ and the differential elliptic flow $v_{2}\left(p_{\mathrm{T}}\right)$ for all charged particles from minimum bias collision events exhibit only minor sensitivity to the shape of the initial energy density profile. Thus, the conclusions extracted from earlier studies of elliptic flow using the wounded nucleon parametrization (which is now disfavoured by the new multiplicity data) remain valid. For identified heavy hadrons such as (anti)protons the differential elliptic flow $v_{2}\left(p_{\mathrm{T}}\right)$ at low $p_{\mathrm{T}}$ is smaller for the binary collision induced initial conditions than for the wounded nucleon parametrizations. This stems from the somewhat stronger radial flow created by the "hard" initializations, which should also be visible in the single-particle spectra.

In closing we note that the initialization models studied in the present paper assume a one-to-one correspondence between impact parameter, number of participating nucleons, and initial energy density profile in the transverse plane. This neglects the possibility of strong event-by-event fluctuations in the initial density profile at fixed number of charged particles in the final state. As pointed out recently [37], strong event-by-event fluctuations in the initial energy density profile may result in sizeable event-by-event fluctuations of $v_{2}$ at fixed impact parameter. Our present study shows that small and smooth variations in the initial energy density profile at fixed impact parameter cause effects on the particle multiplicity and on the radial and elliptic flow which compensate each other in such a way that $v_{2}\left(n_{\mathrm{ch}} / n_{\max }\right)$ is almost unaffected. It would be interesting to study whether this compensation also survives the much larger fluctuations studied in Ref. 37].
Acknowledgements: We thank P.V. Ruuskanen for several useful discussions. The work of P.K. was supported in part by the Deutsche Forschungsgemeinschaft. P.H. acknowledges financial support by the Director, Office of Science, Office of High Energy and Nuclear Physics, Division of Nuclear Physics, and by the Office of Basic Energy Sciences, Division of Nuclear Sciences, of the U.S. Department of Energy under Contract No. DE-AC03-76SF00098. K.J.E. and K.T. acknowledge financial support from the Academy of Finland.

[1] J.-Y. Ollitrault, Phys. Rev. D 46 (1992) 229.

[2] S.A. Voloshin and Y. Zhang, Z. Phys. C 70 (1996) 665.

[3] H. Sorge, Phys. Rev. Lett. 78 (1997) 2309; ibid. 82 (1999) 2048.

[4] B. Zhang, M. Gyulassy, and C.M. Ko, Phys. Lett. B 455 (1999) 45.

[5] P.F. Kolb, J. Sollfrank and U. Heinz, Phys. Lett. B 459 (1999) 667; P.F. Kolb, J. Sollfrank, P.V. Ruuskanen, and U. Heinz, Nucl. Phys. A 661 (1999) 349.

[6] P.F. Kolb, J. Sollfrank, and U. Heinz, Phys. Rev. C 62 (2000) 054909.

[7] K.H. Ackermann et al. (STAR Collaboration), Phys. Rev. Lett. 86 (2001) 402.

[8] P.F. Kolb, P. Huovinen, U. Heinz, and H. Heiselberg, Phys. Lett. B 500 (2001) 232.

[9] P. Huovinen, P.F. Kolb, U. Heinz, P.V. Ruuskanen, and S.A. Voloshin, Phys. Lett. B 503 (2001) 58.

[10] Presentations by the STAR, PHENIX, and PHOBOS collaborations in [11].

[11] Proceedings of "Quark Matter 2001" (Stony Brook, Jan. 15-20, 2001), to appear in Nucl. Phys. A. Transparencies of the contributions are available at http:// www.rhic.bnl.gov/qm2001/program.html.

[12] D. Teaney, J. Laurent, and E.V. Shuryak, nucl-th/ 0011058 .

[13] M. Gyulassy, I. Vitev, and X.N. Wang, Phys. Rev. Lett. 86 (2001) 2537.

[14] X.N. Wang, Phys. Rev. C 63 (2001) 054902.

[15] K.J. Eskola, V.J. Kolhinen and P.V. Ruuskanen, Nucl. Phys. B 535 (1998) 351; K.J. Eskola, V.J. Kolhinen and C.A. Salgado, Eur. Phys. J. C 9 (1999) 61.

[16] K.J. Eskola, K. Kajantie, P.V. Ruuskanen, and K. Tuominen, Nucl. Phys. B 570 (2000) 379.

[17] K.J. Eskola, K. Kajantie, K. Tuominen, Phys. Lett. B 497 (2001) 39.

[18] B.B. Back et al. (PHOBOS Collaboration), Phys. Rev. Lett. 85 (2000) 3100.

[19] K. Adcox et al. (PHENIX Collaboration), Phys. Rev. Lett. 86 (2001) 3500.

[20] G. Roland for the PHOBOS Collaboration, in [11].

[21] M.M. Aggarwal et al. (WA98 Collaboration), Eur. Phys. J. C 18 (2001) 651.

[22] A. Bialas, M. Bleszynski, and W. Czyz, Nucl. Phys. B 111 (1976) 461.

[23] J.-P. Blaizot and J.-Y. Ollitrault, in Quark-Gluon Plasma (R.C. Hwa, ed.), Directions in High Energy Physics, Vol. 6, p. 393 (World Scientific, Singapore, 1990). 
[24] D. Kharzeev, C. Lourenço, M. Nardi, and H. Satz, Z. Phys. C 74 (1997) 307.

[25] D. Teaney and E.V. Shuryak, Phys. Rev. Lett. 83 (1999) 4951.

[26] K. Kajantie, P.V. Landshoff, and J. Lindfors, Phys. Rev. Lett. 59 (1987) 2527; K.J. Eskola, K. Kajantie, and J. Lindfors, Nucl. Phys. B 323 (1989) 37.

[27] K.J. Eskola and K. Kajantie, Z. Phys. C 75 (1997) 515.

[28] K.J. Eskola, P.V. Ruuskanen, S.S. Räsänen and K. Tuominen, hep-ph/0104010.

[29] K.J. Eskola and K. Tuominen, Phys. Rev. D 63 (2001) 114006.

[30] X.-N. Wang and M. Gyulassy, Phys. Rev. Lett. 86 (2001) 3496.

[31] J. Sollfrank et al., Phys. Rev. C 55 (1997) 392.

[32] L. Van Hove, Z. Phys. C 21 (1983) 93.

[33] D. Kharzeev and M. Nardi, nucl-th/0012025, Phys. Lett. $\mathrm{B}$, in press.

[34] M. Gyulassy and T. Matsui, Phys. Rev. D 29 (1984) 419.

[35] A. Dumitru and M. Gyulassy, Phys. Lett. B 494 (2000) 215.

[36] K. Adcox et al. (PHENIX Collab.), nucl-ex/0104015.

[37] T. Osada et al., nucl-th/0102011. 